\title{
(RE)CONOCIENDO EL PATRIMONIO UNIVERSITARIO: EXPERIENCIAS DIGITALES ENTRE ARTE Y MEDIACIÓN
}

\author{
(RE)CONHECENDO O PATRIMÔNIO UNIVERSITÁRIO: EXPERIÊNCIAS \\ DIGITAIS ENTRE ARTE E MEDIAÇÃO
}

David Ruiz Torres

Universidad de Granada/PPGA-UFES/CAPES-PNPD

\begin{abstract}
Resumen: Presentamos una propuesta de valorización del patrimonio universitario a través de las tecnologías digitales móviles que tiene como principal protagonista las obras plásticas inmuebles del campus de Goiabeiras de la Universidade Federal do Espírito Santo (Bra-sil). La edición de una app de geolocalización y realidad aumentada adaptada al entorno del campus será una primera fase de aproximación a las posibilidades de mediación del m-learning a través de sus potencialidades y desafíos que serán analizados en el siguien-te texto.
\end{abstract}

Palavras clave: patrimonio, universidad, digital, arte, mediación.

Resumo: Apresentamos uma proposta de valorização do patrimônio universitário mediante o uso das tecnologias digitais móveis, tendo como principal protagonista as obras plásticas do campus de Goiabeiras da Universidade Federal do Espírito Santo (Brasil). A edição de um aplicativo de geolocalização e realidade aumentada adaptado ao espaço do campus, será a primeira fase de abordagem das possibilidades de mediação do m-learning, tanto nas potencialidades como nos desafios, que serão analisadas no texto a seguir.

Palavras-chave: patrimônio, universidade, digital, arte, mediação. 


\section{Introducción}

Con casi dos décadas a nuestras espaldas del reciente siglo XXI, no podemos sino cerciorarnos de que nos encontramos inmersos en una cultura de la movilidad si consideramos la forma de relacionarnos con nuestro entorno que se halla mediatizado por los dispositivos portátiles y por la ubiquidad del acceso a Internet: en todos los lugares y veinticuatro horas al día.

En esta cultura de la movilidad el gran flujo de informaciones existente a través de las grandes redes digitales, se torna individual a través de los dispositivos portátiles como los smartphones o tablets que permiten que toda esa información sea interiorizada y personalizada a niveles nunca antes conseguidos, y que además esa información sea compartida y distribuida de forma exponencial.

En este contexto de redes informacionales móviles, la educación también ha jugado un papel importante enfrentándose a importantes cambios que han hecho que la experiencia pedagógica sea un acto más allá de la sala de aula y que transcienda tanto en espacios formales, no formales e informales, hablamos del m-learning o aprendizaje móvil.

Para dar una definición más precisa del término $m$-learning debemos considerar que se refiere a una práctica educativa que se ha resumido bajo las palabras de "aquí y ahora" (here and now); un aprendizaje que se produce cuando los alumnos tienen acceso a la información en cualquier momento y en cualquier lugar a través de tecnologías móviles para realizar actividades relacionadas intrínsecamente con el contexto de su aprendizaje (MARTIN y ERTZBERGER, 2013, p. 77).

Por su parte, y no menos importante en el contexto de la educación y el conocimiento, en esta cultura de la movilidad también debemos considerar los usuarios de estos nuevos dis- positivos que son tanto nativos digitales como inmigrantes digitales. Éstos últimos han sabido adaptarse a los nuevos contextos de aprendizaje mediatizados por las nuevas tecnológicas móviles, pues, como nos decía Prensky (2001), los nativos digitales están acostumbrados a recibir información muy rápido y se hallan inmersos en procesos de aprendizajes paralelos y multitarea que son justamente los que caracterizan el m-learning.

\section{M-learning en contextos patrimoniales}

Ya se ha escrito mucho sobre el uso de las nuevas tecnologías digitales móviles (códigos $\mathrm{QR}$, geolocalización, realidad aumentada, etc...) en la educación patrimonial y en la interpretación del objeto cultural mediante aplicaciones y guías multimedia que sirven para visitar tanto espacios museísticos como contextos urbanos y enclaves arqueológicos o patrimoniales in situ.

Y es que en nuestra sociedad del siglo XXI, basada en la proliferación de dispositivos móviles y la conectividad de redes digitales ubicua, se espera una interpretación del patrimonio basada en experiencias de aprendizaje in situ que permitan un conocimiento expandido a través del medio digital.

En la propuesta que presentamos en este texto pretendemos destacar la importancia del patrimonio universitario compuesto por un conjunto de bienes inmuebles y muebles que son custodiados y gestionados por la institución. Este patrimonio universitario tiene un importante valor en el contexto de la comunidad universitaria pero también para la sociedad local que durante los últimos años ha sido objetivo principal para la integración efectiva en el entramado cultural que la institución ofrece (museos, exposiciones, conferencias, teatro, espectáculos audiovisuales, etc...).

Es por ello que la labor de protección y salva- 
guarda del patrimonio universitario ha tenido especial relevancia en los últimos años en una labor de divulgación e interpretación donde las tecnologías digitales han tenido un protagonismo significativo. A este respecto podemos citar algunos ejemplos como el Projeto Resgate do Patrimônio Histórico e Cultural da UFRGS'1 (Universidade Federal do Rio Grande do Sul, Brasil) o el proyecto Atalaya3D² (Patrimonio de las Universidades Andaluzas, España) que no han dudado en aprovechar las posibilidades de la tecnologías digitales para conseguir mejorar la visibilidad del patrimonio universitario.

En este escenario debemos considerar también la importancia pedagógica de las mediaciones tecnológicas ya que nos encontramos en un contexto de enseñanza superior como es la universidad que debe sacar el máximo partido de las tecnologías más punteras en relación a la comunidad universitaria que siempre es una de las más exigentes (RUIZ, 2002).

Es por ello que las tecnologías digitales móviles se plantean como un recurso válido para la interpretación del patrimonio artístico universitario, de la misma forma que los proyectos desarrollados para el patrimonio histórico urbano (GREVTSOVA, 2016), consiguiendo inventariar y proporcionar la información de una manera más comprensible.

Además, las posibilidades de las tecnologías digitales móviles que mencionábamos anteriormente, nos ofrecen una serie de ventajas que favorecen el conocimiento mediante nuevas dinámicas de acceso a la información (multimedia), interactividad y comunicación asociadas al m-learning.

1 https://www.ufrgs.br/patrimoniohistorico/o-que-e/ 2 http://atalaya3d.ugr.es/

\section{(Re)conociendo el patrimonio universitario Punto de partida}

Bajo estas premisas, nuestro ensayo se presenta como una propuesta de valorización del patrimonio universitario que tiene como principal protagonista los bienes artísticos inmuebles de la Universidade Federal do Espírito Santo. La idea surge con la premisa de que para valorizar el legado cultural de cada comunidad o pueblo, el elemento básico es dar a conocer su importancia y llevar a cabo su difusión, con el fin de que los individuos se identifiquen con su propio patrimonio.

Para este ensayo nos centraremos específicamente en las obras plásticas repartidas por el campus de Goiabeiras (Vitória-ES) que en mayor o menor medida están relacionadas con la historia y evolución de la institución y, lo más importante, con la comunidad académica del pasado, presente y futuro.

Como parte de este trabajo, y entendiendo la propuesta como una labor de sensibilización con el patrimonio, las tecnologías digitales son un elemento clave como agentes activos de una mediación que permita a la comunidad universitaria conocer su propio patrimonio.

De esta forma se propone realizar una experiencia de mediación a través de la edición de una app para dispositivos celulares de geolocalización (GeoAumentaty) que permitirá realizar un recorrido por los diferentes ítems artísticos del campus, y que permita acceder a las informaciones sobre los mismos: identificación, historia, autor(es), representatividad dentro de la comunidad universitaria, etc.

\section{Desarrollo de la app}

Inicialmente se realizó un mapeamiento de los monumentos y obras plásticas repartidas por el campus de Goiabeiras de la Universidade Federal do Espírito Santo con la intención de in- 
Figura 1. Imagen de la app GeoAumentaty con el mapeamiento de las obras plásticas inmuebles repartidas por el campus de Goiabeiras - UFES, 2018. (Captura de pantalla).

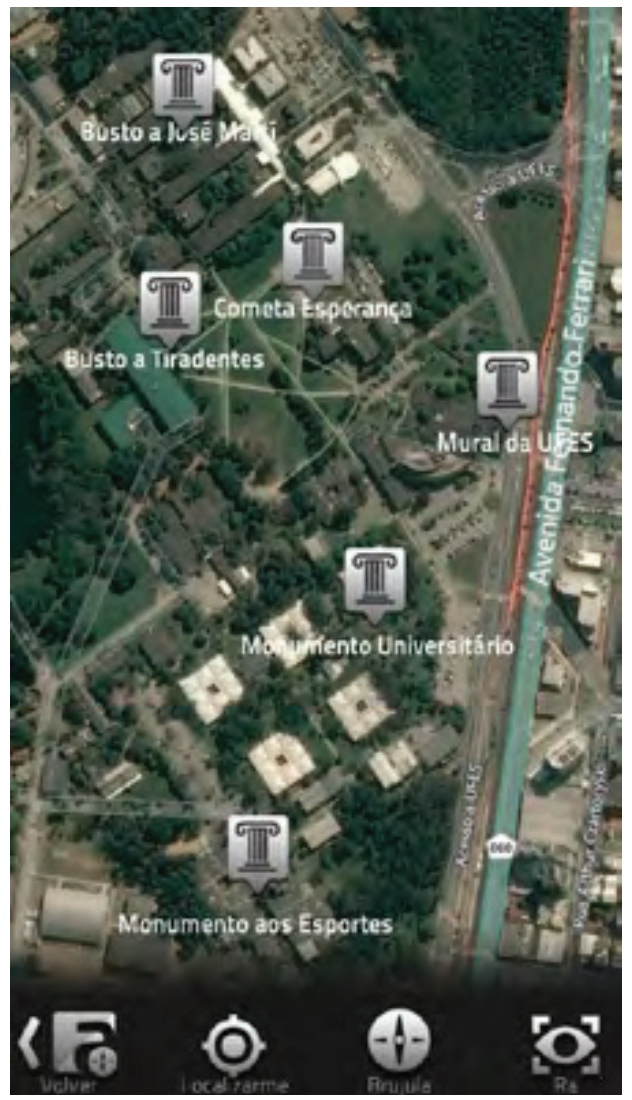

ventariar aquellos ítems que iban a formar parte de los contenidos que contendría la app y tener una primera aproximación (Figura 1). En esta fase se tuvo en cuenta un primer acercamiento a las obras, mediante documentación fotográfica y también en relación a las placas informativas o cartelas conmemorativas que dieron algunas de las pistas (no siempre evidentes o existentes). Posteriormente se hizo necesaria una labor de documentación e investigación que pretendía rescatar el máximo de información posible existente sobre las obras resultantes del mapeamiento a través de las fuentes bibliográficas y hemerográficas, tanto analógicas como digitales, que permitieron obtener los contenidos necesarios para cada una de las obras que serían mostradas a través de la app.
Una vez recabada la información y documentación gráfica se pasó a la edición de la app GeoAumentaty ${ }^{3}$ a través de la web oficial para la que se creó la ruta denominada "Patrimonio UFES" con varios puntos de interés. Esta aplicación para dispositivos portátiles, disponible en las plataformas de contenidos digitales, se basa en la geolocalización y la realidad aumentada de forma que permite construir un mapa interactivo del campus de Goiabeiras en el que se localizan los POIs (Point Of Information) que indican las obras plásticas repartidas por el espacio universitario. De esta forma se introdujeron un total de siete POIs (Figuras 2 y 3 ) correspondientes a los ítems seleccionados previamente después de la exploración in situ que fueron:

- Busto de Alaor de Queiroz Araújo

- Busto de José Martí

- Busto de Tiradentes

- Cometa Esperança

- Monumento a los Deportes

- Monumento Universitario

- Mural de la UFES

Las posibilidades de la app son varias pues las diferentes rutas pueden realizarse en base a la exploración libre de los usuarios o, de otro modo, establecer un recorrido tipo gymkana con una secuencia de POIs a seguir.

Así, cada POls estaba señalado en el mapa a través de un icono tras el que se accedía a la ficha técnica y las informaciones adicionales sobre la obra en cuestión. Al habilitar la visión con realidad aumentada (RA) permitía usar la cámara del dispositivo para buscar en el espacio circundante los iconos y la distancia a la que se encontraban desde la posición del usuario.

Además se acompañaba de un álbum fotográfico con diferentes puntos de vista de la obra o detalles significativos de la misma. Otros re-

3 http://geo.aumentaty.com/info/ 


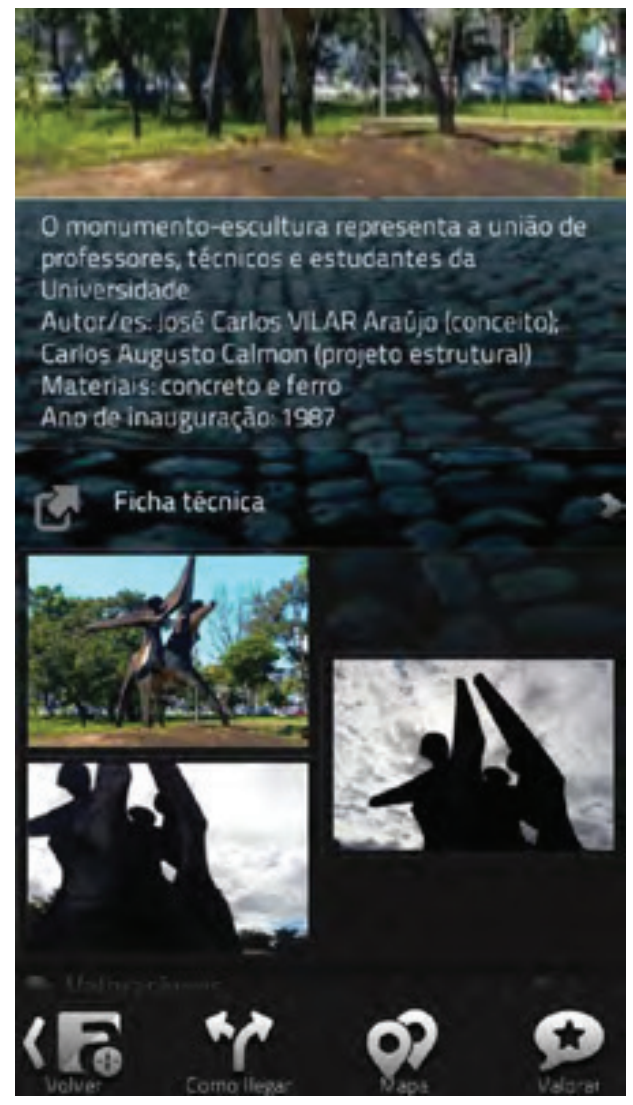

cursos multimedia (PFD, vídeo, etc...) también formaron parte de la documentación extra que podía visionarse a través de la app en relación a cada una de las obras.

\section{Desafíos y futuras líneas de ensayo}

A pesar de que comentamos las posibilidades de aprendizaje propias del m-learning existen una serie de desafíos que deben ser tenidos en cuenta desde varias perfectivas pero fundamentalmente desde la tecnológica y la educacional. Es por ello por lo que debemos considerar algunas limitaciones y debilidades propias de los dispositivos móviles que ya fueron consideradas por algunos autores (KUKULSKA-HULME, 2007) y que se refieren a:

- Funcionalidad limitada de los dispositivos

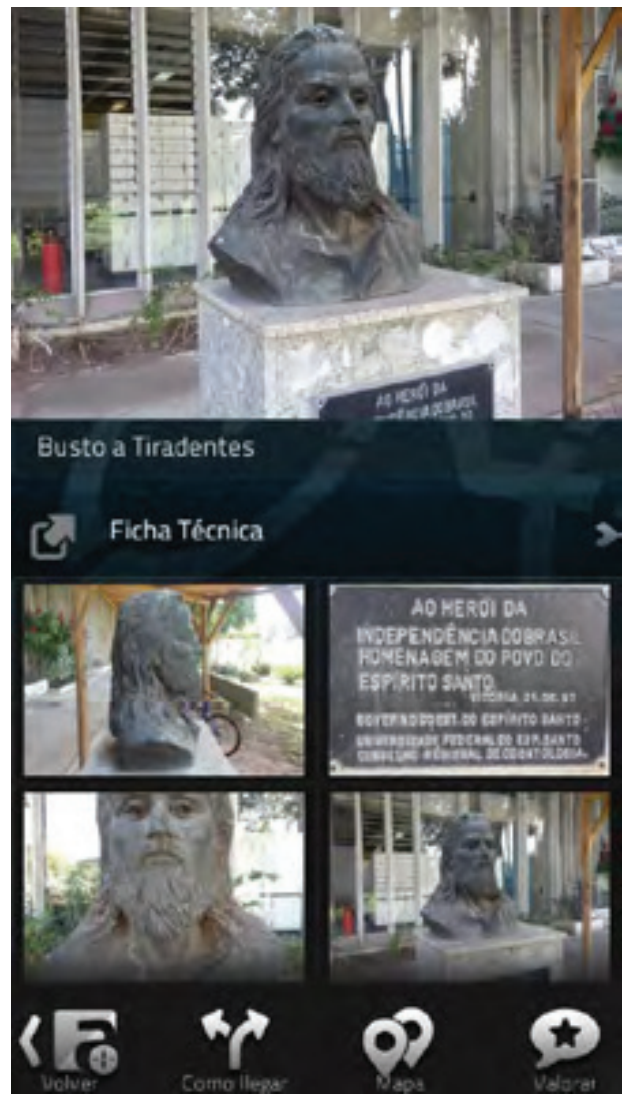

de los usuarios como tamaño de la pantalla o memoria obsoleta que pueden perjudicar aprendizaje.

- Fallos de programación propios del software escogido que impedirían una correcta dinámica en la consulta y navegación por los contenidos;

- Exigencia de una conexión móvil a Internet para el correcto funcionamiento de la app que depende del proveedor de la red y la disponibilidad de la misma por parte del usuario;

- las condiciones ambientales del espacio al aire libre no se encuentran controladas como por ejemplo ocurriría en la sala de un museo: brillo de la pantalla, condiciones meteorológicas adversas, etc.

Todo ello afectaría directamente a una correcta experiencia educativa a través de la me-
Figuras 2 y 3 . Ejemplos de la app GeoAumentaty con las obras contenidas y las informaciones adicionales de interés para los usuarios, 2018. (Captura de pantalla). 
todología del e-learning, siendo factores que podrían variar los juicios de valoración de la app por parte de los usuarios.

Por su parte, también debemos considerar que el aprendizaje móvil aún no presenta un marco teórico sólido que pueda servir de guía para la consecución de un diseño instructivo efectivo y evaluar la calidad de las aplicaciones que dependen de las tecno logías digitales móviles (PARK, 2011, p. 83).

Por este motivo, establecer unos juicios de valor será una fase fundamental de nuestro ensayo ya que la evaluación de los contenidos de la app por parte de los usuarios será clave para evidenciar la pertinencia de las tecnologías digitales móviles y el e-learning como una forma válida de interpretación, conocimiento y valoración del patrimonio y, específicamente, del patrimonio universitario de la Universidade Federal do Espírito Santo. Es por ello que la próxima etapa de desarrollo de este ensayo cuenta con un proceso de evaluación y valoración por parte de los miembros de la comunidad universitaria que se enfrentarán a los contenidos de la app y a una encuesta sobre su conocimiento del patrimonio universitario.

\section{Conclusiones}

El patrimonio universitario se muestra como parte de la memoria material e inmaterial de la historia de la institución y, lo más importante, con la comunidad académica del pasado, presente y futuro. A pesar de esto, la interpretación y conocimiento de este patrimonio universitario a veces se dibuja sombría y encontramos ciertas dificultades para transmitir los valores de este patrimonio a la comunidad universitaria debido a agentes de puesta en valor precarios.

Se espera que esta primera fase de la investigación proporcione una importante herramienta para la interpretación del patrimonio univer- sitario in situ, que, a través de las tecnologías digitales móviles como instrumento de mediación, permita el conocimiento y difusión para la comunidad universitaria.

Por último, lo que se pretende con este estudio es llegar a una concienciación sobre el patrimonio universitario del campus para una intervención precisa sobre su mantenimiento y estado de conservación actual que, en muchos de los casos que recogemos en la app, se encuentran en la necesidad de una intervención de restauración y plan preventivo anual.

\section{Referencias}

GREVTSOVA, Irina. Tendencias del uso de las tecnologías móviles en espacios urbanos: m-learning y patrimonio cultural. revista $\mathrm{PH}, \mathrm{n}^{\circ}$ 90, pp. 132-151, 2016.

KUKULSKA-HULME, Agnes. Mobile usability in educational contexts: what have we learnt? The international review of research in open and distributed learning, 2007, vol. 8, n².

MARTIN, Florence; ERTZBERGER, Jeffrey. Here and now mobile learning: An experimental study on the use of mobile technology. Computers \& Education, 2013, vol. 68, pp. 76-85.

PARK, Yeonjeong. A pedagogical framework for mobile learning: Categorizing educational applications of mobile technologies into four types. The International Review of Research in Open and Distributed Learning, 2011, vol. 12, no 2, pp. 78-102.

PRENSKY, Marc. Digital natives, digital immigrants part 1. On the horizon, 2001, vol. 9, no 5, pp. 1-6.

RUIZ, Guillermo. La sociedad del conocimiento y la educación superior universitaria. Revista mexicana de ciencias políticas y sociales, 2002, vol. 45, no 185. 